\title{
Transverse multibunch instabilities for two bunch trains on separated orbits
}

\author{
B. C. Jiang, ${ }^{1}$ G. X. Xia, ${ }^{2}$ M. Z. Zhang, ${ }^{1}$ S. Q. Tian, ${ }^{1}$ and Z. T. Zhao ${ }^{1, *}$ \\ ${ }^{1}$ Shanghai Institute of Applied Physics, Chinese Academy of Sciences, Shanghai 201800, China \\ ${ }^{2}$ School of Physics and Astronomy, University of Manchester, \\ Manchester and the Cockcroft Institute, United Kingdom
}

(Received 25 August 2013; published 16 April 2014)

\begin{abstract}
Transverse multibunch instabilities including the resistive wall instability and ion instabilities are investigated analytically and numerically for two bunch trains on separated orbits in a storage ring. The two separated orbits' operating mode appears more stable against transverse multibunch instabilities than the conventional single orbit operating mode and may be beneficial to high current operations for colliders or synchrotron light sources.
\end{abstract}

DOI: 10.1103/PhysRevSTAB.17.040703

PACS numbers: 29.27. $-\mathrm{a}, 29.20 . \mathrm{db}, 42.72 .-\mathrm{g}$

\section{INTRODUCTION}

It has been reported in $[1,2]$ that a camshaft bunch can be produced by a fast kicker at the repetition rate of the revolution frequency of the storage ring at the Advanced Light Source (ALS). The camshaft bunch in the ALS storage ring permanently stays on the distorted orbit so that the radiation is separated from that of the bunch train and can be used for time-of-flight experiments. The technique can be extended to produce two spatially separated bunch trains (TSSB) in one storage ring if the pulse width is stretched to about half of the revolution period and one of the bunch trains is kicked to the distorted orbit by the fast kicker while the other is unaffected. Figure 1 gives a sketch of two separated closed orbits for the Shanghai Synchrotron Radiation Facility (SSRF) storage ring; the one marked in blue is the design orbit (or golden orbit) and the other, marked in red, is the distorted orbit, produced by a kicker located at the end of the second straight section.

The behaviors of transverse multibunch instabilities of the TSSB will be much different from that of a single bunch train. The differences are not only from the orbit departure but also from the amplitude-dependent tune shift. In this paper the transverse resistive wall instability is studied in Sec. II, and the ion instabilities are studied in Sec. III. In Sec. IV we will discuss the potential applications of the TSSB. Some of the side effects such as dynamic aperture shrinkage and transverse coupling increase will also be discussed.

\footnotetext{
zhaozt@sinap.ac.cn

Published by the American Physical Society under the terms of the Creative Commons Attribution 3.0 License. Further distribution of this work must maintain attribution to the author $(s)$ and the published article's title, journal citation, and DOI.
}

\section{RESISTIVE WALL INSTABILITY}

\section{A. Tune separation effects}

The bunch train on the distorted orbits will introduce amplitude-dependent tune shifts.

For the SSRF cases, the vertical tune shift produced by the vertical kicker is shown in Fig. 2. Two bunch trains, one on the golden orbit and the other on the distorted orbit, will have a vertical tune separation, which in turn will affect the resistive wall instability in the storage ring.

To avoid the complexity of the analytical treatment of the instability for the nonsymmetric filling pattern [3], we assume that the first bunch train contains only one bunch and the second bunch train contains uniformly filled bunches around the ring. The equation of the betatron oscillations with the driving force of the transverse wake field (assuming that the wake field generated by the first single bunch train can be neglected) can be written as

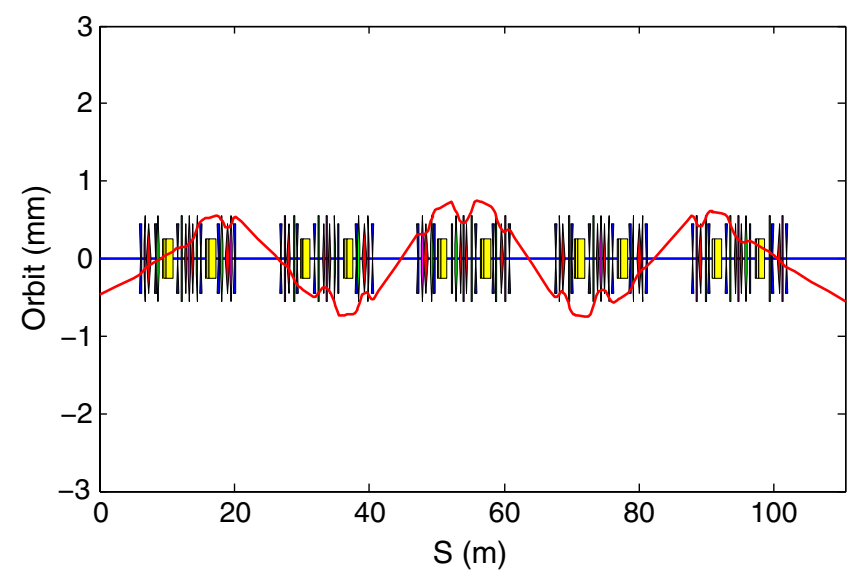

FIG. 1. Sketch of the two spatially separated bunch trains in the SSRF storage ring. (A quarter of the full ring lattice is shown here. The blue line is the golden orbit and the red one is the distorted orbit that is kicked by $100 \mu \mathrm{rad}$ in the vertical plane by a fast kicker located at the end of the second straight section.) 


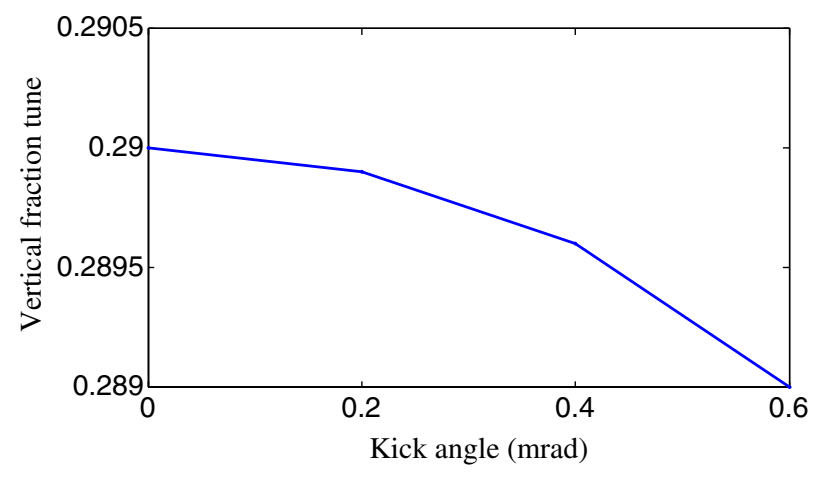

FIG. 2. Vertical tune shift produced by the vertical kicker (located at the end of the second straight section) at the SSRF storage ring.

$$
\begin{aligned}
\ddot{y}_{1}+ & 2 \alpha \dot{y}_{1}+\omega_{\beta 1}^{2} y_{1} \\
= & -\frac{c r_{e} N}{T_{0} \gamma} \sum_{k} \sum_{m=0}^{M-1} W_{\perp}\left(-k C-\frac{m-1}{M} C\right) y_{m} \\
& \times\left(t-k T_{0}-\frac{m-1}{M} T_{0}\right),
\end{aligned}
$$

Here $\alpha$ is the radiation damping rate, $M$ is the bunch number in the second bunch train, $c$ is the speed of light, $N$ is the number of electrons in one bunch, $T_{0}$ the revolution period, $\gamma$ the relativistic energy, $W_{\perp}$ the transverse wake field, $r_{e}$ the classical electron radius, and $C$ the circumference of the storage ring.

Assume that the second bunch train has the oscillation pattern

$$
y_{m}^{\mu}(t)=A \times \exp \left(2 \pi \frac{m \mu}{M} i\right) \exp \left(-i \Omega_{\mu} t\right)
$$

with $\mu=0,1,2, \ldots M-1$. By substituting Eq. (2) into Eq. (1) we get

$$
\begin{aligned}
\ddot{y}_{1} & +2 \alpha \dot{y}_{1}+\omega_{\beta 1}^{2} y_{1} \\
& \left.=i A \frac{r_{e} N 4 \pi M}{T_{0} \gamma Z_{0} T_{0}} \sum_{p=-\infty}^{\infty} Z_{\perp}\left[(p M+\mu) \omega+\omega_{\beta}\right)\right] \exp \left(-i \Omega_{\mu} t\right),
\end{aligned}
$$

where $Z_{0}$ is the impedance of the vacuum. Eq. (3) is a forced vibration equation, and the solution can be written as

$$
y_{1}=F * \exp \left(-i \Omega_{u} t-\psi\right),
$$

with

$$
\begin{array}{r}
F=A \frac{\left.i \frac{r_{e} N 4 \pi M}{\gamma T_{0}^{2} Z_{0}} \sum_{p=-\infty}^{\infty} Z_{\perp}\left[(p M+\mu) \omega+\omega_{\beta}\right)\right]}{\left[\left(\Omega_{\mu}^{2}-\omega_{\beta 1}^{2}\right)^{2}+2 \alpha^{2} \Omega_{\mu}^{2}\right]^{1 / 2}} \\
\approx A \frac{\left.i \frac{r_{e} N M}{\gamma T_{0} Z_{0} v_{\beta}} \sum_{p=-\infty}^{\infty} Z_{\perp}\left[(p M+\mu) \omega+\omega_{\beta}\right)\right]}{\alpha / \sqrt{2}},
\end{array}
$$

and

$$
\psi=a \tan \frac{2 \alpha \Omega_{\mu}}{\omega_{\beta 1}^{2}-\Omega_{\mu}^{2}} .
$$

Equation (4) shows that for a forced vibration, the first single bunch train will experience a steady state oscillation, and the amplitude will not grow exponentially. The real part of the numerator in Eq. (5) is proportional to the instability growth rate of the second bunch train divided by the radiation damping rate.

The two-bunch train interaction will not develop an instability from this simplified analytical treatment. The instability growth rate depends only on its own bunch train current which means that the resistive wall instability threshold will be doubled for the TSSB.

\section{B. Offset effects}

To evaluate the effects of transverse offsets of the two bunch trains, the equation of the betatron oscillations (neglecting radiation damping) is rewritten as

$$
\begin{aligned}
\ddot{y}_{n}+\omega_{\beta}^{2} y_{n}= & -\frac{c r_{e} N}{T_{0} \gamma} \sum_{k}\left\{\sum_{m=0}^{K} W_{\perp}\left(-k C-\frac{m-n}{M} C\right)\right. \\
& \times\left[y_{m}\left(t-k T_{0}-\frac{m-n}{M} T_{0}\right)-\Delta y_{1}\right] \\
& +\sum_{m=K+1}^{M-1} W_{\perp}\left(-k C-\frac{m-n}{M} C\right) \\
& \left.\times\left[y_{m}\left(t-k T_{0}-\frac{m-n}{M} T_{0}\right)-\Delta y_{2}\right]\right\} .
\end{aligned}
$$

Here $K$ is the bunch number in the first bunch train. $\Delta y_{1,2}$ are the offsets of the orbits for the first and second bunch trains. After rearrangment, the equation can be written in the following form:

$$
\begin{aligned}
\ddot{y}_{n}+\omega_{\beta}^{2} y_{n}= & -\frac{c r_{e} N}{T_{0} \gamma}\left\{\sum_{k} \sum_{m=0}^{M-1} W_{\perp}\left(-k C-\frac{m-n}{M} C\right) y_{m}\right. \\
& \left.\times\left(t-k T_{0}-\frac{m-n}{M} T_{0}\right)-\Delta F\right\}
\end{aligned}
$$




$$
\begin{aligned}
\Delta F= & \sum_{k}\left[\sum_{m=0}^{K} W_{\perp}\left(-k C-\frac{m-n}{M} C\right) \Delta y_{1}\right. \\
& \left.+\sum_{m=K+1}^{M-1} W_{\perp}\left(-k C-\frac{m-n}{M} C\right) \Delta y_{2}\right] .
\end{aligned}
$$

Here the following assumptions are made:

$$
y_{n}^{\mu}=A * \exp \left(2 \pi \frac{n \mu}{M} i\right) \exp \left(-i \Omega_{\mu} t\right)+f(t),
$$

and

$$
\omega_{\beta 1} \approx \omega_{\beta 2} \approx \Omega_{\mu}
$$

The solution of Eq. (7) can be written in the following form:

$$
\begin{gathered}
\left.\Omega_{\mu}-\omega_{\beta}=-i \frac{r_{e} N M}{\gamma T_{0} Z_{0} v_{\beta}} \sum_{p=-\infty}^{\infty} Z_{\perp}\left[(p M+\mu) \omega+\omega_{\beta}\right)\right], \\
f(t)=-\frac{\Delta F}{\omega_{\beta}^{2}} .
\end{gathered}
$$

The result shows that the resistive wall instability of the TSSB is the same as for the single-bunch train case with an additional static term that is created by the orbit offsets. The static term does not contribute to any instability and could be compensated by the vertical correctors.

\section{Tracking of the resistive wall instability}

The analytical results derived above are based on several assumptions. The actual interaction between the two bunch trains of the TSSB will be more complicated than the analytical model. To obtain more accurate results, a tracking code was developed to evaluate the resistive wall instability.

In the tracking code, the electron bunches are represented by macroparticles that are run through the ring by manipulating a transfer matrix:

$$
\left[\begin{array}{cc}
\cos \left(v_{y} \pi\right)+\alpha \sin \left(v_{y} \pi\right) & \beta \sin \left(v_{y} \pi\right) \\
-\gamma \sin \left(v_{y} \pi\right) & \cos \left(v_{y} \pi\right)+\alpha \sin \left(v_{y} \pi\right)
\end{array}\right] .
$$

Here $\alpha, \beta, \gamma$ are the vertical Twiss parameters. The values are chosen at a place where $\beta=\langle\beta\rangle$. The bunches then receive a transverse wake field kick as follows [4]:

$$
d y_{\text {wake }}^{\prime}=-\frac{e V_{\perp}}{\gamma m_{0} c^{2}}=-\frac{e}{\gamma m_{0} c^{2}} \frac{2 L}{\pi b^{3}} F \sqrt{\frac{c}{\sigma}} \sum_{n} \frac{N e y_{n}}{\sqrt{z_{n}}} .
$$

Here $e$ is the electron charge, $m_{0}$ is the mass of the electron, $L$ is the length of the chamber, $b$ is the equivalent chamber radius, $F$ is a shape factor, $\sigma$ is the conductivity of the chamber metal, and $y_{n}, z_{n}$ denote previous bunch transverse coordinate and distance from the present bunch, respectively.

To keep the tracking to a reasonable duration, the wake fields within 80 turns are recorded to calculate the transverse kick force. The total number of bunches are 100 and 50 for each respective train. Three cases are tracked for comparison. One case is two trains, both on the golden orbit. The other two cases are one of the bunch trains is kicked by $200 \mu \mathrm{rad}$ and by $400 \mu \mathrm{rad}$, respectively. The instability thresholds for the three cases are 80, 120, and $130 \mathrm{~mA}$ as shown in Fig. 3. Although the resistive wall instability threshold of the TSSB cannot be doubled
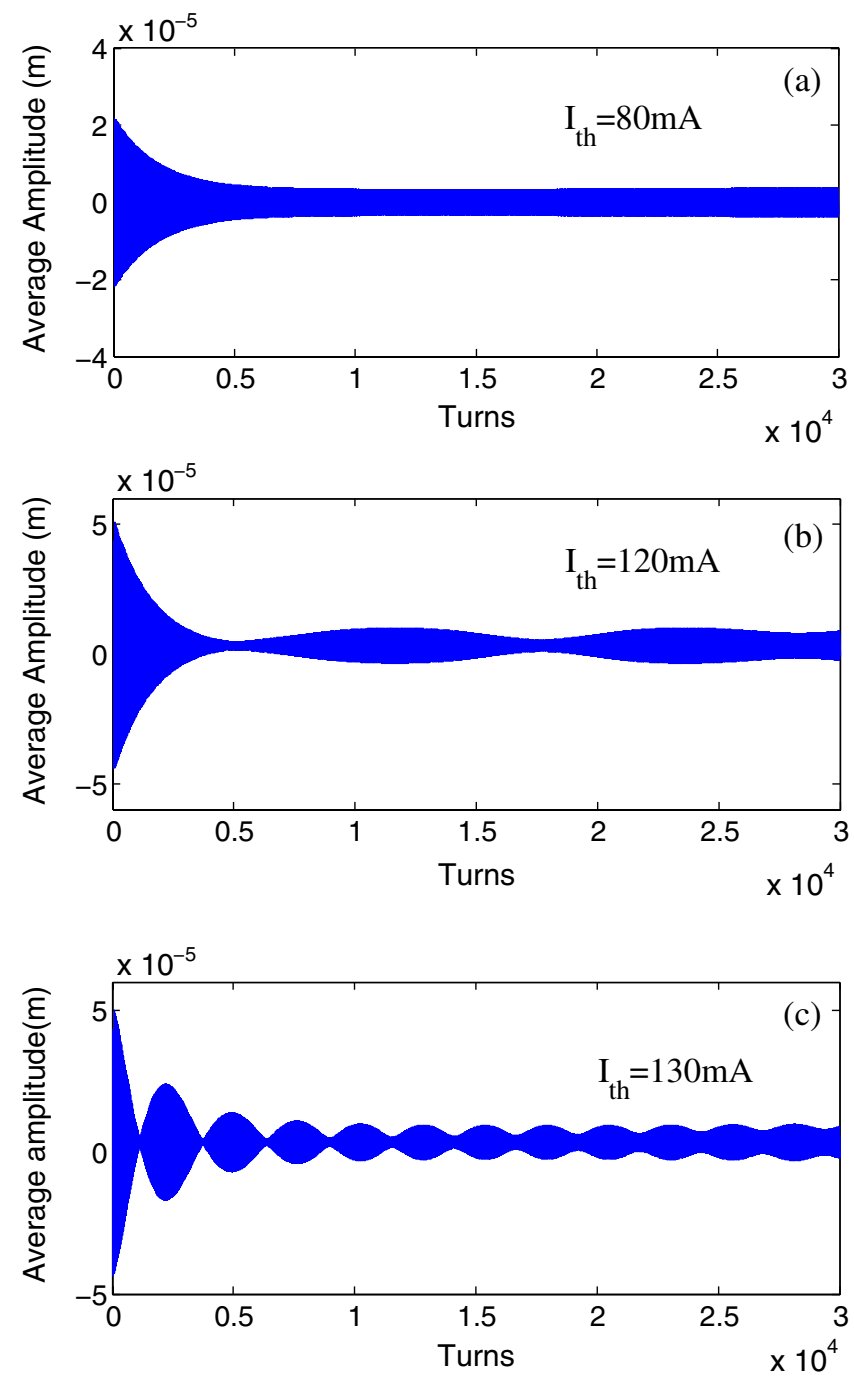

FIG. 3. Tracking results showing the resistive wall instability for three different cases. (a) average beam oscillation amplitude of two bunch trains on the golden orbit; (b) average amplitude of two bunch trains with $200 \mu \mathrm{rad}$ separation; (c) average amplitude of two bunch trains with $400 \mu \mathrm{rad}$ separation. The amplitude approaching a constant value indicates that an instability threshold has been found. 
compared to the single beam operation mode as predicted by the analytical result, the increment is considerable and is around $50 \%$.

\section{BEAM ION INSTABILITIES}

\section{A. Ion trapping}

To address the ion trapping effect for the TSSB, a simulation study has been performed. For a Gaussian bunch, the transverse potential well produced by the electron bunch can be written as [5]

$$
V(x, y)=-\frac{N}{\pi \varepsilon_{0}} \int_{0}^{\infty} \frac{1-e^{-\frac{x^{2}}{2 \sigma_{x}^{2}+4 t} \frac{y^{2}}{2 \sigma_{y}^{2}+4 t}}}{\sqrt{\left(2 \sigma_{x}^{2}+4 t\right)\left(2 \sigma_{y}^{2}+4 t\right)}} d t .
$$

Here $\sigma_{x}$ and $\sigma_{y}$ are rms transverse beam sizes, and $\varepsilon_{0}$ is the permittivity of free space. For a $2 \mathrm{D}$ treatment we derive $E(y)$ at $x=0$

$$
\begin{aligned}
E(y) & =\left.\frac{\partial V(x, y)}{\partial y}\right|_{x=0} \\
& =-\frac{N}{\pi \varepsilon_{0}} \int_{0}^{\infty} \frac{1-2 e^{-\frac{y^{2}}{2 \sigma_{y}^{2}+4 t}} y}{\sqrt{\left(2 \sigma_{x}^{2}+4 t\right)}\left(2 \sigma_{y}^{2}+4 t\right)^{3 / 2}} d t .
\end{aligned}
$$

Neglecting the longitudinal motion of the ion, when an electron bunch passes by the transverse kick to the ion is

$$
\Delta \dot{y}=-\frac{2 r_{p} c N}{A} \int_{0}^{\infty} \frac{2 e^{-\frac{y^{2}}{2 \sigma_{y}^{2}+4 t}}\left(y-y_{e}\right)}{\sqrt{\left(2 \sigma_{x}^{2}+4 t\right)}\left(2 \sigma_{y}^{2}+4 t\right)^{3 / 2}} d t .
$$

Here $A$ is the mass number of the ion.

A simulation code was developed to investigate the motion of the ions. The kick to the ion produced by the electron bunch is calculated by numerically evaluating the integral. The consequences of the integral term in Eq. (18) are shown in Fig. 4. In the region $y \ll \sigma_{y}$, it is approximately linear and matches the expansion formula [6]

$$
\Delta \dot{y} \propto \frac{2 y}{\sigma_{y}\left(\sigma_{y}+\sigma_{x}\right)} .
$$

In the code, the kick strength is calculated using an interpolation method according to the instantaneous $y$ value. The interpolation table is that used for plotting Fig. 4. Between two adjacent kicks is a $2 \mathrm{~ns}$ free drift space. In our simulation, 500 ion macroparticles are created randomly in one $\sigma_{y}$.

The beam parameters at the observation point are listed in Table I. For different kinds of operating modes the simulation results are shown in Fig. 5.

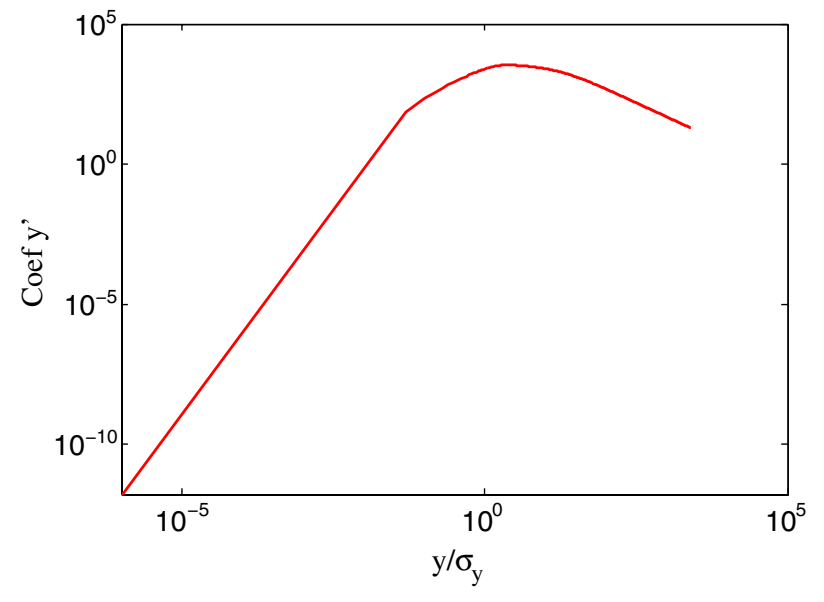

FIG. 4. The integral term in Eq. (18) as a function of $y / \sigma_{y}$.

At different observation points (different orbit departure and beam size), we obtain different results; however, the overall tendency is maintained. The results show that the TSSB helps to drive the ions to large amplitudes in a finite number of turns, which will reduce the possibility of ion trapping and therefore weaken the instability.

\section{B. Fast ion instability}

The fast ion instability (FII) has been observed in many electron storage rings $[7,8]$, and is caused by the linearly increasing density of the ions along the bunch train. It is a single passage phenomenon, the number of ions produced by a long bunch train is large enough to disturb the motion of the trailing bunches and thus increase the oscillation amplitudes and the beam size. After passage of the bunch train most of the ions are lost during the empty gap.

The FII is simulated by a weak-strong simulation code [9]. In this simulation, the beam is regarded as a rigid Gaussian beam and the ions produced by the collisional ionization are represented by macroparticles. The beam parameters used are the same as those used in the ion trapping simulation.

The vertical oscillation amplitude of the bunch centroid is characterized by the square root of the Courant-Snyder invariant $J_{y}=\left[\gamma y^{2}+2 \alpha y y^{\prime}+\beta y^{\prime 2}\right] / 2$. For case (c) in Fig. 5, the growth of $J_{y}$ is about two orders smaller than case (b) in Fig. 5 as shown in Fig. 6.

TABLE I. Basic beam parameters of the SSRF storage ring for ion trapping calculation.

\begin{tabular}{lc}
\hline \hline Parameters & Value \\
\hline Beam size $\sigma_{x} / \sigma_{y}[\mu \mathrm{m}]$ & $158 / 10$ \\
Number of electrons per bunch & $0.54 \mathrm{e} 10$ \\
rf frequency $[\mathrm{MHz}]$ & 500 \\
Ion type & $\mathrm{CO}+$ \\
Orbit separation $(y$ plane) $[\mu \mathrm{m}]$ & 200 \\
\hline \hline
\end{tabular}



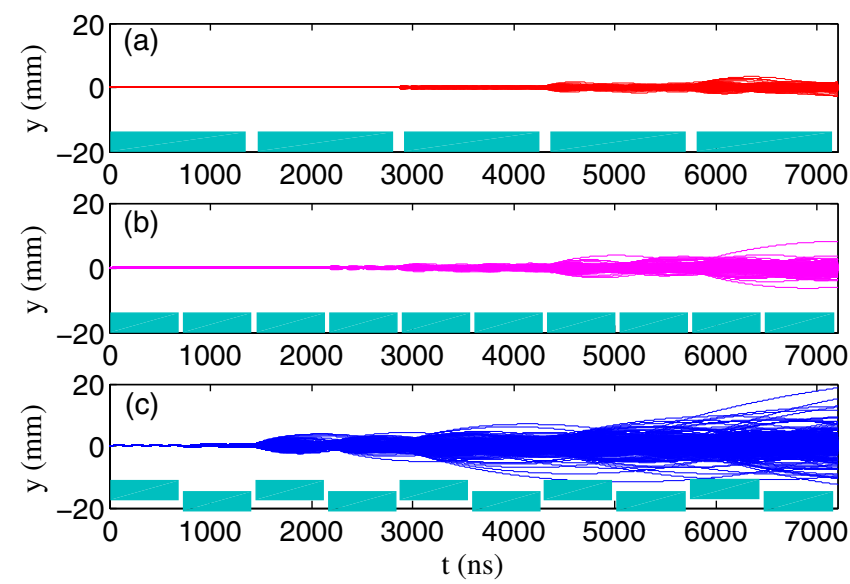

FIG. 5. The oscillation amplitude of ions with respect to the circulation time in the SSRF storage ring, tracking 5 turns. (a) 1 bunch train with 700 bunches followed by a 20 bunch empty gap; (b) 2 bunch trains with 350 bunches each, separated by a 10 bunch empty gap; (c) two spatially separated bunch trains of 350 bunches each, separated by a 10 bunch empty gap, run at different closed orbits with displacement $200 \mu \mathrm{m}$ at the observation point.

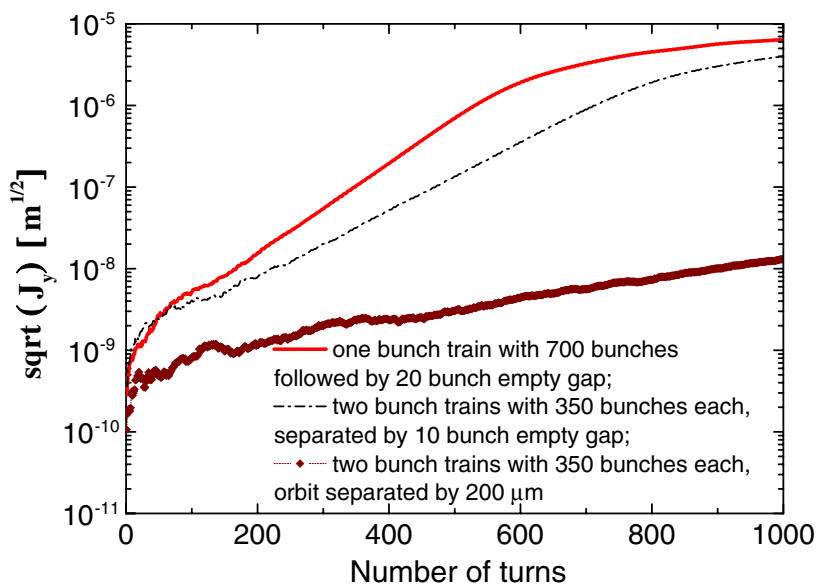

FIG. 6. Growth of the maximum oscillation amplitude of the electron bunch centroid for the same parameters used in the ion trapping simulation in Fig. 5.

\section{DISCUSSIONS}

The treatment of wake field in the analytical method in Sec. II is general, which can be directly applied to the instability caused by the transverse higher order mode (HOM) of the rf cavity. A more detailed tracking had been performed with SSRF superconducting (SC) rf cavity transverse HOM parameters as listed in Table II. In the tracking, after electron bunch receives the resistive wall wake field kick as indicated in Eq. (15), a HOM kick is added [10]:

$$
d y^{\prime}=-\frac{e}{\gamma m_{0} c^{2}} \sum_{n} \sum_{m} \operatorname{real}\left(i N e y_{n} \frac{R_{\perp m} \omega_{m}}{Q_{m}} e^{\left(i \omega_{m}-\frac{\omega_{m}}{2 Q_{m}} \frac{z_{n}}{c}\right.}\right) .
$$

TABLE II. Transverse HOM parameters of SSRF CESR type SC cavity (with ferrite absorbers).

\begin{tabular}{lcr}
\hline \hline Frequency $(\mathrm{MHz})$ & $\mathrm{R}_{\perp} / \mathrm{Q}(\mathrm{Ohm} / \mathrm{m})$ & $\mathrm{Q}$ \\
\hline 631.993 & 5.55 & 70 \\
631.994 & 5.54 & 70 \\
673.998 & 23.41 & 46 \\
674.001 & 23.41 & 46 \\
\hline \hline
\end{tabular}

Since the HOM of the SC cavity is highly damped, the major wake field contribution is from a resistive wall. The simulation results vary little compared to Fig. 3; for the same three cases the threshold is 79,119 , and $129 \mathrm{~mA}$, respectively.

For the TSSB, the study of single particle beam dynamics shows that there are very limited degradations of the beam performance. The shrinkage of the dynamic aperture is shown if Fig. 7, and is small enough that it may have no effect on the injection efficiency and beam lifetime.

The increasing transverse coupling for a $400 \mu \mathrm{rad}$ kicked beam for the SSRF case is about $0.8 \%$ which is also acceptable for operation.

Application of TSSB in storage-ring-based light sources will produce two spatially separated photon beams from one source point such as a wiggler or an undulator, an approach that may potentially double the number of available beam line stations. The suppression of the instabilities in the TSSB makes it possible to achieve a higher current to make the photon brightness of each beamlet comparable to the single-bunch train operation mode if rf power is not limited. To achieve this, many technical challenges need to be solved such as fast kicker strength, vacuum chamber design, beam line optics arrangement, and so on.

Although the study is based on the light source storage ring, the application of TSSB seems more feasible for colliders. If two rings both operate in the TSSB scheme, and collide at the interaction point for each pair of closed orbits, the instability threshold can be greatly improved.

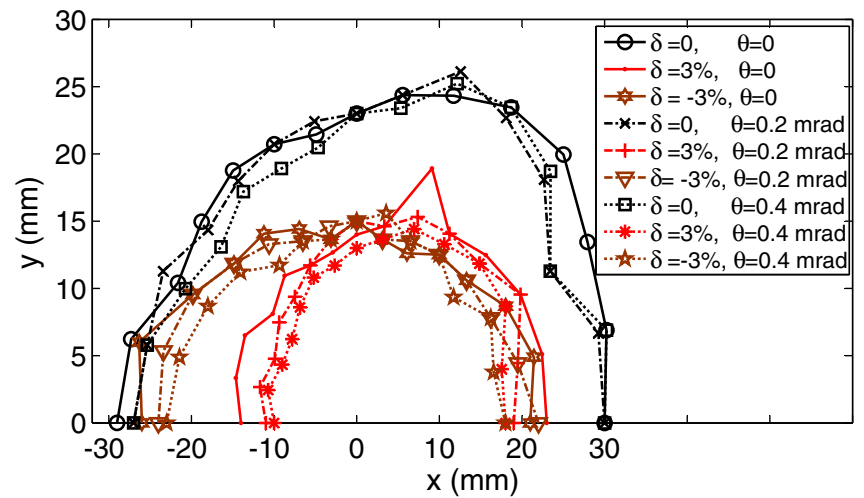

FIG. 7. Dynamic apertures for on-momentum and off-momentum beams for different operating modes (blue lines indicate on-momentum particles and green and red lines indicate offmomentum particles). 
The kick angles for the TSSB in the collider are not necessary as large compared to light sources that will greatly reduce the technical challenges. For positron or proton beams, the benefit of the TSSB to the electron cloud instability is still an open question to be studied.

\section{ACKNOWLEDGMENTS}

The authors would like to thank Dr. J. H. Chen and Dr. C. Jing for useful discussions. This work was supported by the Youth Innovation Promotion Association of CAS and the National Natural Science Foundation of China under Grant No. 11105214.

[1] C. Sun, G. Portmann, M. Hertlein, J. Kirz, and D. S. Robin, Phys. Rev. Lett. 109, 264801 (2012).

[2] G. Portmann, S. Kwiatkowski, J. Julian, M. Hertlein, D. Plate, R. Low, K. Baptiste, W. Barry, and D. Robin, in 2008 Beam Instrumentation Workshop, BIW08 (JACoW, Tahoe City, CA, 2008), pp. 213-217.
[3] Zhao Zhen-Tang, and Jiang Bo-Cheng, High Energy Phys. Nucl. Phys. 28, 1209 (2004).

[4] Jiang Bo-Cheng, Liu Gui-Min, and Zhao Zhen-Tang, High Energy Phys. Nucl. Phys. 31, 956 (2007).

[5] Y.C. Lee and C.S. Hwang, in Proceedings of the European Particle Accelerator Conference, EPAC96, Barcelona, Spain (IOP, Bristol, 1996).

[6] D. Villevald and S. Heifets, Report No. SLAC-TN-06-032, 1993.

[7] B. Jiang, G. Xia, L. Han, G. Liu, Z. Dai, and Z. Zhao, Nucl. Instrum. Methods Phys. Res., Sect. A 614, 331 (2010).

[8] L. Wang, J. Safranek, Y. Cai, J. Corbett, T. Raubenheimer, J. Schmerge, and J. Sebek, in Proceedings of the 2nd International Particle Accelerator Conference, IPAC2011, San Sebastián, Spain (EPS-AG, Spain, 2011), pp. 814-816.

[9] Guoxing Xia, Bocheng Jiang, and Guimin Liu, in Proceedings of the International Particle Accelerator Conference, IPAC-10, Kyoto, Japan (ICR, Kyoto, 2010), pp. 1991-1993.

[10] M. J. Byrd, in Proceedings of the Particle Accelerator Conference, PAC-95, Dallas, TX (IEEE, New York, 1995), pp. 2684-2686. 